\title{
The Utility of Spleen Stiffness Measurement in Grading the Severity of Esophageal Varices in Cirrhotic Patients
}

\author{
Seno $\mathrm{SM}^{1}$, Ira Inductivo-Yu${ }^{1 *}$, Calimag $\mathrm{AP}^{1}$ and Silva $\mathrm{GM}^{2}$ \\ ${ }^{1}$ Division of Internal Medicine, National Kidney and Transplant Institute, \\ Philippines \\ ${ }^{2}$ Division of Radiology, National Kidney and Transplant Institute, Philippines \\ *Corresponding Author: Ira Inductivo-Yu, Gastrohepatologist, Division of Internal \\ Medicine, National Kidney and Transplant Institute, Philippines.
}

Received: January 23, 2021

Published: February 27, 2021

(C) All rights are reserved by Ira Inductivo-Yu., et al.

\begin{abstract}
Introduction: Bleeding esophageal varices is a complication of liver cirrhosis with significant morbidity/mortality. Upper gastrointestinal endoscopy for variceal screening upon diagnosis of cirrhosis is recommended, however expense and invasiveness limit its use. A reproducible, non-invasive surrogate marker to screen for varices is needed. Spleen Stiffness can predict the presence and severity of varices with high accuracy. However, local data determining cut-off values of spleen stiffness in grading varix severity is lacking.

Objectives: This study aims to determine cut-off values of spleen stiffness that correlate with esophageal varix severity.

Materials and Methods: A retrospective analytic cohort study was done, analyzing 48 liver cirrhosis patients who underwent Spleen Elastography and endoscopy to evaluate for esophageal varices. Variables that showed significant difference proceeded to post hoc analysis (Duncan). The relationships between the parameters were characterized using Spearman's correlation coefficients.

Results: Spleen Stiffness was higher in patients with varices. All groups were comparable in demographics, laboratory findings, ascites, MELD and Child Pugh Class. Variceal grades I and II were higher in malignancy; grades III and IV showed higher spleen size. Grade III varices showed significantly higher stiffness. Cut-offs for Grade I, II, III varices were 6.22, 6.90, and 10.01, respectively. A cutoff value for Grade IV varices could not be set $(p=0.436)$. A significant correlation was found for both spleen size and stiffness, but stiffness was found to be more predictive of variceal grade.

Conclusion: Spleen elastography is a cost-effective, non-invasive alternative to variceal screening and should be considered in cirrhotic patients.
\end{abstract}

Keywords: Cirrhosis; Elastography; Esophageal Varix; Portal Hypertension; Spleen Stiffness

\section{Abbreviations}

MELD: Model for End Stage Liver Disease; PH: Portal Hypertension; EV: Esophageal varices; HVPG: Hepatic Venous Portal Gradient; BEV: Bleeding Esophageal Varices; SS: Spleen Stiffness; LSM: Liver Stiffness Measurement; SSM: Spleen Stiffness Measurement;
HRV: High Risk Varices; NKTI: National Kidney and Transplant Institute

\section{Introduction}

Liver cirrhosis is the final evolutive stage of any chronic liver disease and its clinical outcomes are modulated by the degree of

Citation: Ira Inductivo-Yu., et al. "The Utility of Spleen Stiffness Measurement in Grading the Severity of Esophageal Varices in Cirrhotic Patients". Acta Scientific Gastrointestinal Disorders 4.3 (2021): 50-60. 
portal hypertension. It is estimated to be responsible for over one million deaths worldwide, affecting an estimated $2 \%$ of the global population [1]. Portal hypertension (PH) is a frequent complication of cirrhosis, contributing to the development of esophageal varices (EV) [2]. The hepatic venous portal gradient (HVPG) is the standard used to determine the degree of $\mathrm{PH}$ and is found to correlate with the presence of EV [3]. An HVPG >10 mmHg predicts the presence of $\mathrm{EV}$, while a value $>12 \mathrm{mmHg}$ is predictive for variceal bleeding [4]. PH results in splenic congestion and increased spleen stiffness. It also induces architectural changes in the splenic arteries and veins and induces fibrosis [5].

Bleeding esophageal varices (BEV) is a life-threatening event with a $10-20 \%$ mortality with each episode [6]. Due to the high pervasiveness of varices and the significant morbidity associated with variceal hemorrhage, early recognition of clinically significant esophageal varices has been the subject of many scientific inquiries. However, in clinical practice, portal hypertension and esophageal varices are evaluated mainly thru invasive procedures requiring specialized training and specialty units, either by endoscopy or hepatic vein catheterization.

Recent guidelines by the American Association for the Study of Liver Diseases recommend that all cirrhotic patients undergo screening endoscopy at diagnosis to identify varices and those who warrant primary prophylaxis against hemorrhage [5]. However, invasive testing is potentially associated with complications, related to sedation and the procedure itself, expensive and time-consuming [7]. Additionally, only 15 - 25\% of patients subject to screening endoscopy were found to have medium/large varices requiring prophylactic therapy [8]. Therefore, there is a need for a non-invasive surrogate marker for the presence and severity of esophageal varices which is simple, objective, reproducible and accurate.

\section{Review of Related Literature}

Studies have found that a strong correlation was observed between clinically significant HVPG and spleen stiffness (SS), and in cases where SS measurement (SSM) was used as a screening test for the indication of esophagogastroduodenoscopy, only one of 100 screened patients would have wrongly avoided esophagogastroduodenoscopy (EGD) [9]. In a local study done by Calimag., et al. [11], a total of 29 subjects underwent spleen elastography and they found a strong positive correlation of spleen stiffness and severity of esophageal varices $(r=0.821)$ (i.e. spleen stiffness was increased in patients with endoscopically larger varices), but a lesser degree of correlation between spleen diameter and severity of esophageal varices $(r=0.446)$ [11]. The same study also concluded that spleen size did not produce a significant difference in between groups of variceal size (implying that it may not be accurate in predicting and monitoring esophageal varix progression), but the lack of effect may be due to the small sample size utilized in the study [11].

The splenic changes observed in patients with portal hypertension is not simply attributed to passive spleen congestion, but also to tissue hyperplasia characterized by a combination of angiogenesis, fibrogenesis, enlargement and hyperactivation of splenic lymphoid compartment [12]. These changes may be better observed through elastography and is better reflective of complex hemodynamic changes observed in portal hypertension [3,5]. Hence, the usefulness of splenomegaly and spleen stiffness in the diagnosis of portal hypertension and prediction of esophageal varices has been a matter of recent scientific interest.

SSM can be used to predict the presence and severity of esophageal varices with high degree of accuracy in patients with chronic liver disease. In one study done on Hepatitis C- predominant patients, elastography had a sensitivity of $98.5 \%$ and specificity of $98.9 \%$, respectively [5]. SS predicts the formation of esophageal varices caused by splanchnic hemodynamic changes better than liver stiffness. To date, only 1 local correlational study proving the utility of spleen elastography in predicting the severity of esophageal varices was done, but there is yet to be a local study that determines cut-off values of spleen stiffness in grading esophageal varix severity.

\section{Definition of terms}

1. Spleen stiffness: An ultrasonographic measure, expressed in $\mathrm{kPa}$, reflective of portal hypertension-related changes in the spleen, including splenomegaly.

2. Spleen diameter: The largest dimension of the spleen determined via ultrasonography. Expressed in centimeters.

3. Spleen point shear wave elastography: A non-invasive method proposed for the assessment of splenic fibrosis in patients with chronic liver disease by measuring spleen stiffness via ultrasound.

Citation: Ira Inductivo-Yu., et al. "The Utility of Spleen Stiffness Measurement in Grading the Severity of Esophageal Varices in Cirrhotic Patients". Acta Scientific Gastrointestinal Disorders 4.3 (2021): 50-60. 
4. Portal hypertension: A clinical condition characterized by a high blood pressure in the portal vein and its tributaries and it is defined as a gradient between portal and systemic blood pressure $>6 \mathrm{mmHg}$.

5. MELD (Model of end-stage liver disease) score: Used to estimate relative disease severity and prognosis of patients with chronic liver disease. It is computed using the following parameters: Creatinine, Bilirubin, INR, Dialysis at least twice in the past week.

6. Esophageal varices grading: A method used in this study to quantify esophageal varix severity by size as observed thru endoscopy:

a. Grade I: Small straight esophageal varices.

b. Grade II: Enlarged, tortuous varices occupying less than $1 / 3$ the lumen.

c. Grade III: Large, coil shaped esophageal varices occupying more than $1 / 3$ of the lumen.

d. Grade IV: Racemose varices occluding the lumen, particularly marked with cherry red spots or varices on varices ("cherry red varices").

\section{Research Objectives}

General objectives: To determine cut-off values of spleen stiffness measured by point shear wave elastography in grading esophageal varix severity.

\section{Specific objectives:}

1. To determine the demographic profile of Liver Cirrhosis patients at a tertiary referral center.

2. To determine the spleen stiffness as measured by point shear wave elastography in known cirrhotic patients at a tertiary referral center.

3. To determine the presence and severity of esophageal varices among patients with Liver Cirrhosis at a tertiary referral center.

4. To determine cut-off values of spleen stiffness that correlate with esophageal varix severity.

\section{Research Question}

What cut off value for spleen stiffness via spleen elastography may be used to grade esophageal varix severity?

\section{Materials and Methods}

Study design: A retrospective analytic cohort study.

Study setting and time period: Conducted from August 2018 to March 2020 at a tertiary referral center that caters to challenging liver patients and liver transplantation.

Study population and sample size: All Liver Cirrhosis Patients, regardless of cause, seen at NKTI from August 2018 to March 2020 were included. Both outpatients or inpatients were included in the study. Patients with previous EGD and Spleen Elastography were enrolled. A non-probability convenience sampling method was employed.

Inclusion criteria:

1. All Liver Cirrhosis Patients above 18 years old, regardless of cause of cirrhosis.

2. Patients diagnosed with liver cirrhosis made by a combination of clinical, biochemical (platelet count, international normalized ratio, prothrombin time, alanine aminotransferase [ALT], albumin, bilirubin), and radiographic imaging (Ultrasound or CT Scan; Liver size and Characteristics).

3. All patients underwent upper GI endoscopy and Spleen Elastography.

\section{Exclusion criteria:}

1. Patients who did not undergo both procedure or underwent only one of the specified procedures.

2. Presence of portal vein thrombosis on imaging.

3. Those patients who were hemodynamically unstable to undergo endoscopy.

\section{study procedure}

All study participants had a previous EGD and spleen elastography done as out/inpatient. The principal investigator conducted a chart review of the patients in the study. Diagnostics/imaging/ endoscopy reports were reviewed from the inpatient chart at the medical records or outpatient chart from the attending physician's clinic. Consent for review of the chart was taken from the chief of the medical records division and attending physician.

\section{Statistical analysis}

Descriptive statistics was used to summarize the demographic 
The Utility of Spleen Stiffness Measurement in Grading the Severity of Esophageal Varices in Cirrhotic Patients

and clinical characteristics of the patients. Frequency and proportion were used for categorical variables. Mean and SD were used for normally distributed continuous variables. Chi Square test (for categorical variables) and t-test (for continuous variables) were used to determine the difference between liver cirrhotic patients with and without varices in terms of demographic and clinical characteristics of the patients. Kruskal Wallis (for categorical variables) and One way ANOVA (for continuous variables) were used to determine the difference among the different grades of esophageal varices in terms of demographic and clinical characteristics of the patients. Variables that showed significant difference were further subjected to post hoc analysis (Duncan) to determine subsets. The relationships between the parameters were characterized using Spearman's correlation coefficients. Missing variables were neither replaced nor estimated. Null hypotheses were rejected at 0.05 $\alpha$-level of significance. SPSS version 17.0 was used for data analysis.

\section{Ethical considerations}

Informed consent, confidentiality and security of information

Permission and approval from the Institutional Ethics and Review Board (IERB) were obtained prior to the start of the study. Before the commencement of data collection, consent was taken from the medical records/ attending physician for review of the patient's chart.

All data gathered were kept confidential and were used only for the purposes of the study. Patients were assigned an alphanumeric code known only to the investigator. The code assigned to each patient was used to track the date and keep the patient anonymous. All personal data were kept in a secure location under lock and key. Confidential information was not shared during the publication of the research.

\section{Results}

A total of 48 patients were enrolled after the 1.6-year period. The baseline clinical, biochemical endoscopic and radiological findings of the study population are summarized in table 1.

\begin{tabular}{|l|c|c|c|c|c|}
\hline \multicolumn{1}{|c|}{ Variable } & n & $\begin{array}{c}\text { Frequency } \\
\text { (\%) }\end{array}$ & Mean & \pm & SD \\
\hline Age (years) & 48 & & 57.65 & \pm & 15.20 \\
\hline Sex & 48 & & & & \\
\hline Male & & $25(52.1 \%)$ & & & \\
\hline Female & & $23(47.9 \%)$ & & & \\
\hline Etiology & 48 & & & & \\
\hline
\end{tabular}

\begin{tabular}{|c|c|c|c|c|c|}
\hline Hep B & & $32(66.7 \%)$ & & & \\
\hline NAFLD & & $9(18.8 \%)$ & & & \\
\hline ALD & & $2(4.2 \%)$ & & & \\
\hline Others & & $5(10.4 \%)$ & & & \\
\hline Malignancy & 48 & $12(25.0 \%)$ & & & \\
\hline \multicolumn{6}{|l|}{$\begin{array}{l}\text { Laboratory } \\
\text { Findings }\end{array}$} \\
\hline ALT (IU/L) & 47 & & 56.66 & \pm & 54.38 \\
\hline AST (IU/L) & 40 & & 96.68 & \pm & 114.49 \\
\hline Albumin (g/dL) & 37 & & 3.22 & \pm & 0.73 \\
\hline $\begin{array}{l}\text { Bilirubin (mg/ } \\
\mathrm{dL} \text { ) }\end{array}$ & 46 & & 4.98 & \pm & 8.21 \\
\hline $\begin{array}{l}\text { Platelet Count } \\
\left(\times 10^{\wedge} 3 / \mathrm{uL}\right)\end{array}$ & 48 & & 145.73 & \pm & 86.81 \\
\hline INR & 46 & & 1.32 & \pm & 0.31 \\
\hline $\begin{array}{l}\text { Creatinine (mg/ } \\
\mathrm{dL} \text { ) }\end{array}$ & 48 & & 1.14 & \pm & 1.45 \\
\hline $\mathrm{Na}(\mathrm{mmol} / \mathrm{L})$ & 47 & & 136.94 & \pm & 6.41 \\
\hline \multicolumn{6}{|l|}{$\begin{array}{l}\text { Radiologic } \\
\text { Findings } \\
\end{array}$} \\
\hline Spleen Size & 48 & & 513.96 & \pm & 551.25 \\
\hline $\begin{array}{l}\text { Spleen Diameter } \\
(\mathrm{cm})\end{array}$ & 48 & & 12.49 & \pm & 3.28 \\
\hline $\begin{array}{l}\text { Ascites on } \\
\text { Imaging }\end{array}$ & 45 & & & & \\
\hline None & & $21(46.7 \%)$ & & & \\
\hline Minimal & & $11(24.4 \%)$ & & & \\
\hline Moderate & & $9(20.0 \%)$ & & & \\
\hline Massive & & $4(8.9 \%)$ & & & \\
\hline $\begin{array}{l}\text { Esophageal } \\
\text { Varices }\end{array}$ & 48 & & & & \\
\hline No Varices & & $14(29.2 \%)$ & & & \\
\hline Grade I & & $10(20.8 \%)$ & & & \\
\hline Grade II & & $10(20.8 \%)$ & & & \\
\hline Grade III & & '8 (16.7\%) & & & \\
\hline Grade IV & & $6(12.5 \%)$ & & & \\
\hline MELD Score & 46 & & 15.24 & \pm & 7.76 \\
\hline $\begin{array}{l}\text { Child Pugh } \\
\text { Score }\end{array}$ & 18 & & 8.39 & \pm & 2.28 \\
\hline $\begin{array}{l}\text { Spleen Stiffness } \\
(\mathrm{kPa})\end{array}$ & 48 & & 11.69 & \pm & 7.61 \\
\hline
\end{tabular}

Table 1: Baseline characteristics of study population with liver cirrhosis. 
The Utility of Spleen Stiffness Measurement in Grading the Severity of Esophageal Varices in Cirrhotic Patients

54

Of 48 patients, majority were male $(n=25,52.1 \%)$. The primary etiologies of the underlying chronic liver disease were Viral Hepatitis B ( $\mathrm{n}=32,66.7 \%)$, Non-Alcoholic Fatty-liver Disease ( $\mathrm{n}=$ 9, 18.8\%), Alcoholic Liver Disease ( $\mathrm{n}=2,4.2 \%)$ and Others comprised as: AIH ( $n=1,2.1 \%)$, Cardiac Cirrhosis ( $n=1,2.1 \%)$, Schistosomiasis ( $n=1,2.1 \%)$, Hepatitis $C(n=1,2.1 \%)$ and Cryptogenic $(\mathrm{n}=1,2.1 \%) .25 \%$ of patients had hepatocellular carcinoma $(\mathrm{n}=$ 12). The mean Spleen diameter was $12.49 \pm 3.28 \mathrm{~cm}$ and Spleen stiffness was $11.69 \pm 7.61 \mathrm{kPa}$. The mean MELD Score was 15.24 \pm 7.76. The mean Child Pugh Score was $8.39 \pm 2.28$. Majority had no ascites on imaging $(n=21,46.7 \%)$. Endoscopic examination revealed varices in 34 patients (71\%) with variceal grade 1 , grade 2 , grade 3 and grade 4 found in 10 (20.8\%), 10 (20.8\%), 8 (16.7\%) and $6(12.5 \%)$, respectively. No varices were seen in 14 patients (29.2\%).

Table 2 provides a summary comparison of clinical characteristics between cirrhotic patients with varices and without varices. No significant difference was observed in age, sex, etiology of cirrhosis, presence of malignancy, laboratory/radiologic findings, MELD score and Child Pugh Score between patients with and without esophageal varices. Spleen Stiffness was significantly higher in patients with esophageal varices compared to those without varices $(p=0.014)$.

\begin{tabular}{|c|c|c|c|c|c|c|c|c|c|}
\hline \multirow{2}{*}{ Variable } & \multicolumn{4}{|c|}{ Without Varices $(n=14)$} & \multicolumn{4}{|c|}{ With Varices $(n=34)$} & \multirow{2}{*}{ p-value } \\
\hline & Frequency (\%) & Mean & \pm & SD & Frequency (\%) & Mean & \pm & SD & \\
\hline Age (years) & & 61.50 & \pm & 12.11 & & 56.06 & \pm & 16.20 & 0.264 \\
\hline Sex & & & & & & & & & 0.145 \\
\hline Male & $5(35.7 \%)$ & & & & $20(58.8 \%)$ & & & & \\
\hline Female & $9(64.3 \%)$ & & & & $14(41.2 \%)$ & & & & \\
\hline Etiology & & & & & & & & & 0.760 \\
\hline Hep B & $10(71.4 \%)$ & & & & $22(64.7 \%)$ & & & & \\
\hline NAFLD & $3(21.4 \%)$ & & & & $6(17.6 \%)$ & & & & \\
\hline ALD & $0(0.0 \%)$ & & & & $2(5.9 \%)$ & & & & \\
\hline Others & $1(7.1 \%)$ & & & & $4(11.8 \%)$ & & & & \\
\hline Malignancy & $2(14.3 \%)$ & & & & $10(29.4 \%)$ & & & & 0.271 \\
\hline \multicolumn{10}{|l|}{ Laboratory Findings } \\
\hline ALT (IU/L) & & 41.36 & \pm & 27.75 & & 63.15 & \pm & 61.58 & 0.212 \\
\hline AST (IU/L) & & 67.50 & \pm & 61.64 & & 106.40 & \pm & 126.71 & 0.359 \\
\hline Albumin (g/dL) & & 3.30 & \pm & 0.89 & & 3.20 & \pm & 0.69 & 0.727 \\
\hline Bilirubin (mg/dL) & & 4.34 & \pm & 6.30 & & 5.26 & \pm & 9.00 & 0.730 \\
\hline $\begin{array}{l}\text { Platelet Count }\left(\mathrm{x} 10^{\wedge} 3 /\right. \\
\mathrm{uL})\end{array}$ & & 133.43 & \pm & 48.11 & & 150.79 & \pm & 98.64 & 0.534 \\
\hline INR & & 1.34 & \pm & 0.37 & & 1.31 & \pm & 0.29 & 0.731 \\
\hline Creatinine (mg/dL) & & 0.91 & \pm & 0.28 & & 1.24 & \pm & 1.71 & 0.477 \\
\hline $\mathrm{Na}(\mathrm{mmol} / \mathrm{L})$ & & 135.64 & \pm & 8.03 & & 137.48 & \pm & 5.65 & 0.374 \\
\hline \multicolumn{10}{|l|}{ Radiologic Findings } \\
\hline Spleen Size & & 320.98 & \pm & 461.86 & & 593.43 & \pm & 571.36 & 0.121 \\
\hline Spleen Diameter (cm) & & 11.093 & \pm & 3.48 & & 13.06 & \pm & 3.06 & 0.057 \\
\hline Ascites on Imaging & & & & & & & & & 0.590 \\
\hline None & $7(53.8 \%)$ & & & & $14(43.8 \%)$ & & & & \\
\hline Minimal & $4(30.8 \%)$ & & & & $7(21.9 \%)$ & & & & \\
\hline Moderate & $1(7.7 \%)$ & & & & $8(25.0 \%)$ & & & & \\
\hline Massive & $1(7.7 \%)$ & & & & $3(9.4 \%)$ & & & & \\
\hline MELD Score & & 14.929 & \pm & 7.25 & & 15.38 & \pm & 8.09 & 0.860 \\
\hline Child Pugh Score & & 9.00 & \pm & 3.61 & & 8.27 & \pm & 2.09 & 0.626 \\
\hline Spleen Stiffness $(\mathrm{kPa})$ & & 7.54 & \pm & 4.45 & & 13.40 & \pm & 8.03 & $0.014^{*}$ \\
\hline
\end{tabular}

Table 2: Comparison of clinical characteristics between cirrhotic patients with and without esophageal varices.

*: Indicates significant difference at alpha 0.05 . 
The Utility of Spleen Stiffness Measurement in Grading the Severity of Esophageal Varices in Cirrhotic Patients

Table 3 summarized the clinical characteristics between cirrhotic patients according to the degree of esophageal varices. All groups were comparable in demographics, clinical profile, laboratory findings, ascites on imaging, MELD score and Child Pugh Class. There was a significant difference among the five groups in terms of malignancy ( $p=0.037)$, spleen size ( $\mathrm{p}=0.032)$, and spleen stiffness $(\mathrm{p}=0.00)$.

\begin{tabular}{|c|c|c|c|c|c|c|c|c|c|c|c|c|c|c|c|c|c|c|c|c|c|}
\hline \multirow[b]{2}{*}{ Variable } & \multicolumn{4}{|c|}{ Without Varices $(n=14)$} & \multicolumn{4}{|c|}{ Grade I $(n=10)$} & \multicolumn{4}{|c|}{ Grade II (n=10) } & \multicolumn{4}{|c|}{ Grade III $(n=8)$} & \multicolumn{4}{|c|}{ Grade IV (n=6) } & \multirow[b]{2}{*}{$\begin{array}{c}\text { p- } \\
\text { value }\end{array}$} \\
\hline & $\begin{array}{c}\text { Fre- } \\
\text { quency } \\
(\%)\end{array}$ & Mean & \pm & SD & $\begin{array}{c}\text { Fre- } \\
\text { quency } \\
(\%)\end{array}$ & Mean & \pm & SD & $\begin{array}{c}\text { Fre- } \\
\text { quency } \\
(\%)\end{array}$ & Mean & \pm & SD & $\begin{array}{c}\text { Fre- } \\
\text { quency } \\
(\%)\end{array}$ & Mean & \pm & SD & $\begin{array}{c}\text { Fre- } \\
\text { quency } \\
(\%)\end{array}$ & Mean & \pm & SD & \\
\hline $\begin{array}{l}\text { Age } \\
\text { (years) }\end{array}$ & & 61.50 & \pm & 12.11 & & 60.10 & \pm & 12.40 & & 58.90 & \pm & 18.41 & & 49.88 & \pm & 14.87 & & 52.83 & \pm & 20.32 & 0.429 \\
\hline Sex & & & & & & & & & & & & & & & & & & & & & 0.515 \\
\hline Male & $\begin{array}{c}5 \\
(35.7 \%)\end{array}$ & & & & $\begin{array}{c}7 \\
(70.0 \%)\end{array}$ & & & & $\begin{array}{c}5 \\
(50.0 \%)\end{array}$ & & & & $\begin{array}{c}4 \\
(50.0 \%)\end{array}$ & & & & $\left(\begin{array}{c}4 \\
(66.7 \%)\end{array}\right.$ & & & & \\
\hline Female & $\begin{array}{c}9 \\
(64.3 \%)\end{array}$ & & & & $\begin{array}{c}3 \\
(30.0 \%)\end{array}$ & & & & $\begin{array}{c}5 \\
(50.0 \%)\end{array}$ & & & & $\begin{array}{c}4 \\
(50.0 \%)\end{array}$ & & & & $\begin{array}{c}2 \\
(33.3 \%)\end{array}$ & & & & \\
\hline Etiology & & & & & & & & & & & & & & & & & & & & & 0.233 \\
\hline Hep B & $\begin{array}{c}10 \\
(71.4 \%)\end{array}$ & & & & $\begin{array}{c}7 \\
(70.0 \%)\end{array}$ & & & & $\begin{array}{c}8 \\
(80.0 \%)\end{array}$ & & & & $\begin{array}{c}5 \\
(62.5 \%)\end{array}$ & & & & $\begin{array}{c}2 \\
(33.3 \%)\end{array}$ & & & & \\
\hline NAFLD & $\begin{array}{c}3 \\
(21.4 \%)\end{array}$ & & & & $\begin{array}{c}2 \\
(20.0 \%)\end{array}$ & & & & $\begin{array}{c}2 \\
(20.0 \%)\end{array}$ & & & & $\begin{array}{c}1 \\
(12.5 \%)\end{array}$ & & & & $\begin{array}{c}1 \\
(16.7 \%)\end{array}$ & & & & \\
\hline ALD & $\begin{array}{c}0 \\
(0.0 \%) \\
\end{array}$ & & & & $\begin{array}{c}0 \\
(0.0 \%)\end{array}$ & & & & $0(0.0 \%)$ & & & & $\begin{array}{c}1 \\
(12.5 \%)\end{array}$ & & & & $\left(\begin{array}{c}1 \\
(16.7 \%)\end{array}\right.$ & & & & \\
\hline Others & $\begin{array}{c}1 \\
(7.1 \%)\end{array}$ & & & & $\begin{array}{c}1 \\
(10.0 \%)\end{array}$ & & & & $0(0.0 \%)$ & & & & $\begin{array}{c}1 \\
(12.5 \%)\end{array}$ & & & & $\begin{array}{c}1 \\
(16.7 \%)\end{array}$ & & & & \\
\hline $\begin{array}{l}\text { Malig- } \\
\text { nancy }\end{array}$ & $\begin{array}{c}2 \\
(14.3 \%)\end{array}$ & & & & $\left(\begin{array}{c}6 \\
(60.0 \%)\end{array}\right.$ & & & & $\begin{array}{c}3 \\
(30.0 \%)\end{array}$ & & & & $\begin{array}{c}0 \\
(0.0 \%) \\
\end{array}$ & & & & $\left(\begin{array}{c}1 \\
(16.7 \%)\end{array}\right.$ & & & & $0.037^{*}$ \\
\hline \multicolumn{22}{|l|}{\begin{tabular}{|l|} 
Labora- \\
tory \\
Findings \\
\end{tabular}} \\
\hline $\begin{array}{l}\text { ALT } \\
(\text { IU /L) }\end{array}$ & & 41.36 & \pm & 27.75 & & 101.20 & \pm & 92.23 & & 50.20 & \pm & 43.08 & & 43.29 & \pm & 25.24 & & 44.50 & \pm & 25.56 & 0.061 \\
\hline $\begin{array}{l}\text { AST } \\
(\mathrm{IU} / \mathrm{L})\end{array}$ & & 67.50 & \pm & 61.64 & & 133.44 & \pm & 166.60 & & 98.20 & \pm & 134.46 & & 118.00 & \pm & 110.35 & & 69.83 & \pm & 56.91 & 0.740 \\
\hline $\begin{array}{l}\text { Albumin } \\
(\mathrm{g} / \mathrm{dL})\end{array}$ & & 3.30 & \pm & 0.89 & & 3.51 & \pm & 0.70 & & 3.19 & \pm & 0.73 & & 2.86 & \pm & 0.61 & & 3.02 & \pm & 0.66 & 0.540 \\
\hline $\begin{array}{l}\text { Bilirubin } \\
\text { (mg/dL) }\end{array}$ & & 4.34 & \pm & 6.30 & & 5.43 & \pm & 10.07 & & 3.66 & \pm & 4.58 & & 4.69 & \pm & 6.32 & & 8.36 & \pm & 15.40 & 0.858 \\
\hline $\begin{array}{l}\text { Platelet } \\
\text { Count } \\
\left(\mathrm{x} 10^{\wedge} 3 /\right. \\
\mathrm{uL})\end{array}$ & & 133.43 & \pm & 48.11 & & 155.60 & \pm & 56.14 & & 141.40 & \pm & 96.95 & & 109.63 & \pm & 66.63 & & 213.33 & \pm & 166.69 & 0.246 \\
\hline INR & & 1.34 & \pm & 0.37 & & 1.30 & \pm & 0.28 & & 1.28 & \pm & 0.32 & & 1.30 & \pm & 0.26 & & 1.36 & \pm & 0.33 & 0.984 \\
\hline $\begin{array}{l}\text { Creati- } \\
\text { nine (mg/ } \\
\text { dL) }\end{array}$ & & 0.91 & \pm & 0.28 & & 1.24 & \pm & 1.19 & & 1.61 & \pm & 2.92 & & 0.79 & \pm & 0.23 & & 1.22 & \pm & 0.89 & 0.763 \\
\hline
\end{tabular}

Citation: Ira Inductivo-Yu., et al. "The Utility of Spleen Stiffness Measurement in Grading the Severity of Esophageal Varices in Cirrhotic Patients". Acta Scientific Gastrointestinal Disorders 4.3 (2021): 50-60. 


\begin{tabular}{|c|c|c|c|c|c|c|c|c|c|c|c|c|c|c|c|c|c|c|c|c|c|}
\hline $\begin{array}{l}\mathrm{Na} \\
(\mathrm{mmol} / \mathrm{L})\end{array}$ & & 135.64 & \pm & 8.03 & & 138.80 & \pm & 6.36 & & 137.60 & \pm & 3.66 & & 139.43 & \pm & 5.19 & & 132.83 & \pm & 6.37 & 0.289 \\
\hline \multicolumn{22}{|l|}{\begin{tabular}{|l|} 
Radio- \\
logic \\
Findings \\
\end{tabular}} \\
\hline $\begin{array}{l}\text { Spleen } \\
\text { Size }\end{array}$ & & 320.98 & \pm & 461.86 & & 352.20 & \pm & 378.25 & & 485.93 & \pm 4 & 417.31 & & 1043.76 & \pm & 646.45 & & 574.20 & \pm & 730.83 & $0.032 *$ \\
\hline $\begin{array}{l}\text { Spleen } \\
\text { Diameter } \\
(\mathrm{cm})\end{array}$ & & 11.09 & \pm & 3.48 & & 11.88 & \pm & 1.96 & & 12.70 & \pm & 3.78 & & 15.11 & \pm & 3.01 & & 12.92 & \pm & 2.60 & 0.079 \\
\hline $\begin{array}{l}\text { Ascites } \\
\text { on Imag- } \\
\text { ing }\end{array}$ & & & & & & & & & & & & & & & & & & & & & 0.125 \\
\hline None & $\begin{array}{c}7 \\
(53.8 \%)\end{array}$ & & & & $\begin{array}{c}1 \\
(11.1 \%)\end{array}$ & & & & $\begin{array}{c}6 \\
(60.0 \%)\end{array}$ & & & & $\begin{array}{c}6 \\
(75.0 \%)\end{array}$ & & & & $\left(\begin{array}{c}1 \\
(20.0 \%)\end{array}\right.$ & & & & \\
\hline Minimal & $\begin{array}{c}4 \\
(30.8 \%)\end{array}$ & & & & $\begin{array}{c}5 \\
(55.6 \%)\end{array}$ & & & & $\begin{array}{c}2 \\
(20.0 \%)\end{array}$ & & & & $\begin{array}{c}0 \\
(0.0 \%)\end{array}$ & & & & $\begin{array}{c}0 \\
(0.0 \%)\end{array}$ & & & & \\
\hline Moderate & $\begin{array}{c}1 \\
(7.7 \%)\end{array}$ & & & & $\begin{array}{c}2 \\
(22.2 \%)\end{array}$ & & & & $\begin{array}{c}1 \\
(10.0 \%)\end{array}$ & & & & $\begin{array}{c}1 \\
(12.5 \%)\end{array}$ & & & & $\left(\begin{array}{c}4 \\
(80.0 \%)\end{array}\right.$ & & & & \\
\hline Massive & $\begin{array}{c}1 \\
(7.7 \%)\end{array}$ & & & & $\begin{array}{c}1 \\
(11.1 \%)\end{array}$ & & & & $\begin{array}{c}1 \\
(10.0 \%)\end{array}$ & & & & $\begin{array}{c}1 \\
(12.5 \%)\end{array}$ & & & & $\begin{array}{c}0 \\
(0.0 \%)\end{array}$ & & & & \\
\hline $\begin{array}{l}\text { MELD } \\
\text { Score }\end{array}$ & & 14.93 & \pm & 7.25 & & 16.60 & \pm & 9.12 & & 13.22 & \pm & 5.56 & & 13.57 & \pm & 7.00 & & 18.67 & \pm & 10.89 & 0.675 \\
\hline $\begin{array}{l}\text { Child } \\
\text { Pugh } \\
\text { Score }\end{array}$ & & 9.00 & \pm & 3.61 & & 7.33 & \pm & 1.63 & & 9.00 & \pm & 2.83 & & 10.00 & \pm & 0.00 & & 8.67 & \pm & 2.42 & 0.742 \\
\hline $\begin{array}{l}\text { Spleen } \\
\text { Stiffness } \\
(\mathrm{kPa})\end{array}$ & & 7.54 & \pm & 4.45 & & 8.33 & \pm & 3.93 & & 10.85 & \pm & 4.70 & & 23.13 & \pm & 8.61 & & 13.13 & \pm & 6.17 & $0.000^{* *}$ \\
\hline
\end{tabular}

Table 3: Comparison of clinical characteristics between cirrhotic patients.

*: Indicates significant difference at alpha $0.05,{ }^{* *}$ : At alpha 0.01 .

Post hoc analysis (Duncan) revealed the following: first, variceal grades I and II were found to be significantly higher in malignancy compared to other grades; second, variceal grades III and IV showed higher spleen size compared to other groups; lastly; a highly significant difference was found between variceal grades, such that grade III varices showed significantly higher stiffness compared to other groups.

Note that the 27 of the 48 spleen sizes were reported as 3-dimensional values $\left(\mathrm{cm}^{3}\right)$, while the rest (21) were 2 - dimensional $\left(\mathrm{cm}^{2}\right)$. To be able to compare them, the volume of the 3-dimensional values and the area of the 2-dimensional values were obtained. This might explain the high SD between spleen sizes. Nevertheless, a significant difference $(p=0.032)$ was found between groups.

Based on the ROC Curve (See figure 1), cut-off values for spleen stiffness and their corresponding sensitivity and specificity were derived, as shown in table 4 and figure 2 . P- values were highly significant $(p<0.01)$ for cut-off values for Grade I, II and III varices; however, since the $p$ value ( $p=0.436)$ was not significant for Grade IV, a cut-off value could not be set for Grade IV varices. A $\mathrm{kPa}$ greater than or equal to 6.22 is $88.2 \%$ sensitive and $64.3 \%$ specific for grade I varices. A kPa greater than or equal to 6.90 is $87.5 \%$ sensitive and $54.2 \%$ specific for grade II varices. A $\mathrm{kPa}$ greater than or equal to 10.01 is $85.7 \%$ sensitive and $70.6 \%$ specific for grade III varices. 

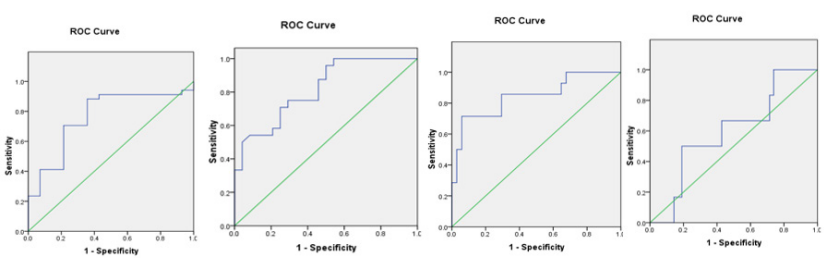

Figure 1: ROC curve for cut-off values of grade 1-4 varices (from left to right).

\begin{tabular}{|l|c|c|c|c|}
\hline & $\begin{array}{c}\text { kPa Cut Off } \\
\text { Value }\end{array}$ & $\begin{array}{c}\mathbf{\%} \\
\text { Sensitivity }\end{array}$ & $\begin{array}{c}\text { \% } \\
\text { Specificity }\end{array}$ & p-value \\
\hline Grade I & 6.22 & 88.2 & 64.3 & $0.005^{* *}$ \\
\hline Grade II & 6.90 & 87.5 & 54.2 & $0.000^{* *}$ \\
\hline $\begin{array}{l}\text { Grade } \\
\text { III }\end{array}$ & 10.01 & 85.7 & 70.6 & $0.000^{* *}$ \\
\hline Grade IV & 10.01 & 66.7 & 57.1 & 0.436 \\
\hline
\end{tabular}

Table 4: kPa cut-off values.

*: Indicates significant difference at alpha $0.05,{ }^{* *}$ : At alpha 0.01 .

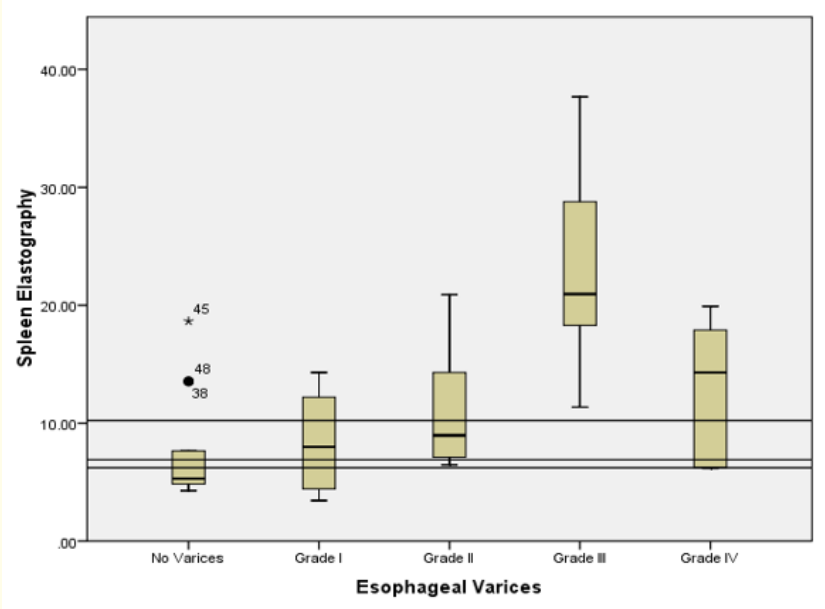

Figure 2: Box plot of kPa cut-off values.

Using Spearman's rho, a positive correlation was found between variceal severity and spleen size $(r=0.339)$ and variceal severity and spleen stiffness $(r=0.554$ ) (See table 5 and figure 3 at the Appendix). As variceal grade increases, an increase in spleen size and stiffness are observed. A significant correlation was found for both spleen size ( $p=0.018)$ and spleen stiffness $(p=0.00)$, but spleen stiffness was found to be more predictive of variceal grade.

\begin{tabular}{|l|c|c|}
\hline \multicolumn{1}{|c|}{ Variable } & r & p-value \\
\hline Spleen Size & 0.339 & $0.018^{*}$ \\
\hline Spleen Stiffness & 0.554 & $0.000^{* *}$ \\
\hline
\end{tabular}

Table 5: Correlation between variceal severity and spleen size/ spleen stiffness.

*: Indicates significant difference at alpha 0.05, **: At alpha 0.01.
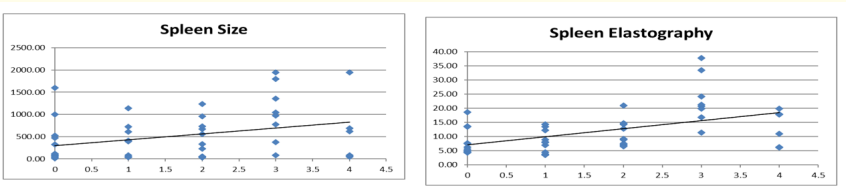

Figure 3: Scatter plot of spleen size/stiffness distribution per variceal grade with best fit line showing correlation.

\section{Discussion}

The outcomes of this study showed that patients with esophageal varices had higher spleen stiffness compared to those patients without varices. In comparison to liver stiffness measurement (LSM) which may be influenced by inflammation, congestion, obstructive jaundice and hepatic blood flow influence which could all simulate "fibrosis" and overestimate values, SS seems able to overcome these factors, making it a more objective marker regardless of the cause [13]. Increased intrasplenic pressure from PH makes it a more accurate predictor of portal pressure [13].

Logically, the higher the esophageal grade, the greater the spleen stiffness $(\mathrm{kPa})$. This was evident in this study where an increasing trend of mean spleen size, diameter and stiffness were observed as the variceal severity increased from None up to Grade III. However, due to the under-representation of patients with grade IV varices ( 6 of 48) and the small sample size utilized in the study, the relatively low mean values for grade 4 was not consistent with 
this upward trend. A similar study by Kim., et al. also found that SS measured by elastography was effective in detecting varices and in predicting the presence of high risk varices [5].

In this study, a higher spleen size did not seem to equate to higher spleen diameter. This might be due to the high SD values between spleen sizes as 27 of 48 values were reported as 3-dimension $\left(\mathrm{cm}^{3}\right)$ and 21 of 48 as 2-dimension $\left(\mathrm{cm}^{2}\right)$; even then, a positive correlation was significant between variceal grade and spleen size.

This study was also able to determine cut-off values that could correlate with esophageal varix severity. However, owing to the small number of cirrhotic patients with Grade 4 varices, a precise cut-off for this grade could not be determined. To date, no recent literature also tried to determined cut-off values for variceal grade severity based on SSM.

Recent literatures cited the Baveno VI guideline to spare screening endoscopies in patients with $\mathrm{LSM}<20 \mathrm{kPa}$ and platelet count $>150 \mathrm{G} / \mathrm{L}$ and rule out high risk varices (HRV) needing treatment [14]. A study done by Colecchia., et al. found that by using SSM as a single diagnostic test to rule out HRV with a single cut-off $\leq 46 \mathrm{kPa}$, the proportion of avoided endoscopies was $35.8 \%$, significantly $(\mathrm{p}<0.01)$ higher than obtained by applying the Baveno VI criteria (21.7\%) [15]. Another study by Stefanescu., et al. combined LSM (cutoff: $19 \mathrm{kPa}$ ) and SSM (cutoff: $55 \mathrm{kPa}$ ) into a simple diagnostic algorithm to rule-in any EV with a sensitivity of 93\% and a PPV of 95\% [10]. The higher cut-off values for SS in the above studies may be due to higher spleen length and volume and height and weight of Caucasians compared to their Asian counterparts [16].

This study also found that lower variceal grades (I and II) were significantly higher in malignancy. Though EV were thought to be more prevalent in advanced Child Pugh Class and hepatocellular cancer (HCCA), making it a marker of poorer prognosis, Giannini., et al's study showed that $56 \%$ of the HCCA patients presented with well-compensated cirrhosis, whereby most varices were small in 352 patients (48.2\% of the EV population) and medium in 220 patients (30.1\%) with HCCA [17]. Whether other factors such as structural/nutritional factors in the HCCA patient contribute to lower variceal grade is uncertain. No other available literature correlated malignancy with variceal grade.

Xing Hu., et al. [18] in their meta-analysis indicated that spleen stiffness measured by current techniques had a fairly good accu- racy for the detection of $\mathrm{PH}$ and EV in CLD patients. In their article, the AUCs for diagnosis of any EV and HREV reached $87 \%$ and $83 \%$, respectively. The results of our study is relatively comparable to this. Furthermore, Xing Hu., et al. observed that the diagnostic performance of SSM for detecting any EV was better across Asian populations than in European populations. This is because prior studies have shown that BMI and central obesity are independent influencing factors for the failure and unreliability of ultrasound in measuring spleen stiffness. The mean BMI of European subjects in other studies was obviously higher than that of Asian subjects. Majority of the patient subjects in our study belonged to the normal BMI range, hence, spleen elastography was performed without too much difficulty.

The limitations of the current study include the small sample size with a mostly homogenous etiologic cause of liver cirrhosis. A larger sample size with wider variety of cirrhosis etiology may produce different results, as SS may be different for esophageal varix prediction amongst different causes of portal hypertension ${ }^{8}$. The cut-off values and findings above are only representative of and/or applicable to the cirrhotic population studied in NKTI.

\section{Conclusion}

In conclusion, the results of this study provide insight into the diagnostic potential of SS in the screening and grading of esophageal varices in patients with liver cirrhosis. SS may prove to be a reliable, non-invasive alternative to endoscopy, especially in the Philippine setting where endoscopy units are scarce and healthcare costs are high. The use of spleen elastography should be considered in patients with cirrhosis as it may help identify those with high risk varices requiring treatment and assist the physician in deciding which patients can safely avoid endoscopy.

\section{Recommendations}

Since cut-off values for Grade IV varices could not be obtained in this study due to the low sample size, a large-scale prospective study including other hospitals in the Philippines may be needed to improve generalizability of the results and cut-off values for spleen stiffness. A larger population size might also show higher diagnostic accuracy of spleen elastography and determination of cut-off value of spleen stiffness for grade IV varices.

The primary investigator recommends a follow-up study incorporating liver stiffness measurement and platelet count with 
spleen stiffness measurement to come up with a prediction model applicable in the Philippine setting to rule out high risk varices.

\section{Acknowledgement}

The author has no conflict of interest and did not receive any funding for this study. The authors would also like to acknowledge the help of Doctor Jade Jamias who have contributed patients in this study, and Doctor Tamana who rendered spleen elastography measurements on some patients.

\section{Bibliography}

1. Tamir BM., et al. "Adverse events of upper GI endoscopy". American Society for Gastrointestinal Endoscopy 76.4 (2012).

2. Berzigotti A., et al. "Elastography, spleen size, and platelet count identify portal hypertension in patients with compensated cirrhosis". Gastroenterology 144.1 (2013): 102-111.

3. Colecchia A., et al. "Measurement of spleen stiffness to evaluate portal hypertension and the presence of esophageal varices in patients with HCV-related cirrhosis". Gastroenterology 143.3 (2012).

4. Dujunco MM., et al. "The Accuracy of Liver Stiffness, Spleen Diameter and Platelet Count Ratio Score (LSPS) Versus Gastroscopy to Predict the Presence of Esophageal Varices in Adult Patients With Liver Cirrhosis". Clinical Gastroenterology and Hepatology 15.1 (2017).

5. Kim HY., et al. "The Role of Spleen Stiffness in Determining the Severity and Bleeding Risk of Esophageal Varices in Cirrhotic Patients". Medicine 94.24 (2015): 1031.

6. Ma X., et al. "Spleen Stiffness Is Superior to Liver Stiffness for Predicting Esophageal Varices in Chronic Liver Disease: A Meta-Analysis". PLoS One 11.11 (2016): e0165786.

7. Mokdad AA., et al. "Liver cirrhosis mortality in 187 countries between 1980 and 2010: a systematic analysis". BMC Medicine 12 (2014): 145.

8. Sharma P., et al. "Spleen Stiffness in Patients with Cirrhosis in Predicting Esophageal Varices". American Journal of Gastroenterology 108.7 (2013): 1101-1107.
9. Singh S., et al. "Accuracy of Spleen Stiffness Measurement in Detection of Esophageal Varices in Patients with Chronic Liver Disease: Systematic Review and Meta-analysis". Clinical Gastroenterology and Hepatology 12.6 (2014): 935-945.

10. Stefanescu H., et al. "Spleen stiffness measurement using Fibroscan for the noninvasive assessment of esophageal varices in liver cirrhosis patients". Journal of Gastroenterology and Hepatology (2020): 164-170.

11. Calimag AP., et al. "A Correlational Study on Spleen Stiffness and the Presence and Severity of Esophageal Varices in Cirrhotic Patients.

12. Rizzo L., et al. "A new sampling method for spleen stiffness measurement based on quantitative acoustic radiation force impulse elastography for noninvasive assessment of esophageal varices in newly diagnosed HCV-related cirrhosis". Biomed Research International (2014): 365982.

13. Roccarina D., et al. "Elastography methods for the non-invasive assessment of portal hypertension". Expert Review of Gastroenterology and Hepatology 12.2 (2017): 155-164.

14. De Franchis F. "Expanding consensus in portal hypertension Report of the Baveno VI Consensus Workshop: Stratifying risk and individualizing care for portal hypertension". EASL Journal of Hepatology 63 (2015): 743-752.

15. Di Biase ML., et al. "A combined model based on spleen stiffness measurement and Baveno VI criteria to rule out high-risk varices in advanced chronic liver disease". Journal of Hepatology 69 (2018): 308-317.

16. Chow KU., et al. "Spleen size is significantly influenced by body height and sex: Establishment of normal values for spleen size at US with a cohort of 1200 healthy individuals". Radiology 279.1 (2016): 306-313.

17. Giannini EG., et al. "Prevalence and prognostic significance of the presence of esophageal varices in patients with hepatocellular carcinoma". Clinical Gastroenterology and Hepatology 4.11 (2006). 
18. Xing Hu., et al. "Diagnostic accuracy of spleen stiffness to evaluate portal hypertension and esophageal varices in chronic liver disease: a systematic review and meta-analysis". European (2020).

\section{Assets from publication with us}

- Prompt Acknowledgement after receiving the article

- Thorough Double blinded peer review

- Rapid Publication

- Issue of Publication Certificate

- High visibility of your Published work

Website: www.actascientific.com/

Submit Article: www.actascientific.com/submission.php

Email us: editor@actascientific.com

Contact us: +919182824667 